\title{
Using Topological Potential Method to Evaluate Node Importance in Public Opinion
}

\author{
Rui SUN \\ Department of Computer Science \\ Chengdu Normal University \\ Chengdu, Sichuan Province, China \\ Key Laboratory of Pattern Recognition and Intelligent \\ Information Processing \\ Institutions of Higher Education of Sichuan Province, \\ Chengdu University \\ Chengdu, Sichuan Province, China \\ e-mail: cug123456@126.com
}

\author{
Wanbo LUO \\ School of Computer Science, Sichuan University \\ Chengdu, Sichuan Province, China \\ e-mail: wbluo@scu.edu.cn
}

\begin{abstract}
The evaluation of node importance is the main research direction in public opinion field, which is much significant to accurately find out the influent nodes for the propagation and evolution of public opinion, furthermore to effective control and predict public opinion situation and in-time guide it. On the basis of the topology structure of network and the attributes of node itself, this paper introduces a method to evaluate node importance in public opinion based on topological potential. Through the theoretical and experimental analysis, it is proved that this method can evaluate the importance of nodes in a fast and accurate way in propagation network of public opinion, which is significant both to theory and practice.
\end{abstract}

Keywords-topological potential; node importance; public opinion; complex networks

\section{INTRODUCTION}

The public opinion is common opinion of public on social phenomenon and social issues [1]. Due to the Internet has fast, flexible merit and other technology characteristics in network transmission, people pay more attention to the research on the spread, monitoring, warning and situation analysis about public opinion in networks.

The evaluation methods of node importance are essentially derived from graph theory and graph-based data mining, focus on the measure about network topology [2]. Social network analysis approaches include degree, closeness, betweeness, information, eigenvector and cumulated nomination, etc [3]. The system science research approach use the connectivity of network to reflect the integrity of certain functions, by measuring the extent of destroyed connectivity of network which caused by removing the nodes to reflect the importance of nodes [4]. The basis of the research is the core and coritivity theory [5].

In the real-world networks, nodes often are not a point on mathematical sense, but are the entities which have many attributes. Especially in propagation network of public opinion, as a node the individual is obviously different, it has the attributes such as the extent of interested in a subject, influenced by others, the activity etc. To some extent, these attributes become the main driver of evolution even more than the network topology [6, 7].

This paper introduces the idea of data field based on complex networks and establishes the evaluation model of node importance based on topological potential in public opinion. Through the theoretical and experimental analysis, it is proved that this method can evaluate node importance in an accurate way, which is significant both to theory and practice.

\section{TOPOLOGICAL POTENTIAL OF NODES}

The topological potential of nodes is based on the concept of data field from cognitive physics [8]. The potential field was used to describe non-contact interactions between matter particles. Taking into account the character of complex networks, we adopt the Gaussian potential function to describe the interaction between nodes, which represent short-range field and has a good mathematical property[9]. The size of the topological potential of nodes describes the value of potential of any nodes, which is effected by itself and neighbors in the network [10].

Definition 1: Given network $G=(V, E)$ with $n$ nodes and $m$ edges, where $V=\left\{v_{1}, v_{2} \ldots, v_{n}\right\}$ denotes set of nodes, $E \subseteq V \times V$ denotes set of edges, and $|E|=m$. According to the potential function definition of data field [10], the topological potential of node $v_{i} \in V$ can be expressed as

$$
\varphi\left(v_{i}\right)=\sum_{j=1}^{n}\left(M_{j} \times e^{-\left(\frac{d_{i j}}{\sigma}\right)^{2}}\right)
$$

Where $d_{i j}$ denotes the distance between $v_{i}$ and $v_{j}$, it is measured by the shortest distance. Impact factor $\sigma$ control the affected area of each node; $M_{j} \geq 0$ denotes the quality of 
node $v_{j}(j=1,2, \ldots, n)$, which is used to describe the attributes of node.

\section{EVAluAtion AlgORITHM OF NODE IMPORTANCE IN PUBLIC OPINION BASED ON TOPOLOGICAL POTENTIAL}

Given the propagation network of public opinion, the description of evaluation algorithm of node importance based on topological potential as follows:

Step1: Computing node quality $M_{j}, j=1,2, \ldots, n$

(1) Building the attribute matrix

The basic attributes of public opinion participants can represents by recognition, debate, influence, obstinacy, activity and the extent which affected by mainstream media, officials, law and so on.

Therefore, we

suppose that $X=\left\{x_{i 1}, x_{i 2}, \ldots, x_{i m}\right\}$ denotes $m$ attributes of node $v_{i}$, where $x_{i j}$ is the $\mathrm{j}$-th attribute of the $\mathrm{i}$-th node. When $f_{i}$ denotes the attribute function, $x_{i j}=f_{i}\left(v_{i}\right), i=1,2, \ldots, n, j=1,2, \ldots, m$. The attribute values of each node constitute the attribute matrix, where $X_{i}, i=1,2, \ldots, m$ denotes $m$ attributes. The attribute matrix is shown in Table 1.

TABLE I. ATtRIBUTE MATRIX

\begin{tabular}{|c|ccccc|}
\hline & $X_{1}$ & $\ldots$ & $X_{j}$ & $\ldots$ & $X_{m}$ \\
\hline$v_{1}$ & $X_{11}$ & $\ldots$ & $x_{1 j}$ & $\ldots$ & $X_{1 m}$ \\
$\cdots$ & $\ldots$ & $\ldots$ & $\ldots$ & $\ldots$ & $\ldots$ \\
$v_{i}$ & $X_{i 1}$ & $\ldots$ & $X_{i j}$ & $\ldots$ & $X_{i m}$ \\
$\cdots$ & $\ldots$ & $\ldots$ & $\ldots$ & $\ldots$ & $\ldots$ \\
$v_{n}$ & $X_{n 1}$ & $\ldots$ & $x_{n j}$ & $\ldots$ & $X_{n m}$ \\
\hline
\end{tabular}

(2) Normalizing the node attribute value

Each element in the attribute matrix is measured by different methods, they have different dimension. In order to comparison and effective evaluation, we should normalize the attribute values and transform them to.

We suppose the original attribute matrix is $X=\left\{x_{i j}\right\}$, the transformed attribute matrix is $Z=\left\{z_{i j}\right\}, i=1,2, \ldots, n$, $j=1,2, \ldots, m, x_{j}^{\max }$ and $x_{j}^{\min }$ are the maximum and minimum in the $\mathrm{j}$-th column of matrix.

$$
z_{i j}=f_{i}\left(v_{i}\right)=\frac{x_{i j}-x_{j}^{\min }}{x_{j}^{\max }-x_{j}^{\min }}
$$

According to Eq.2, we compute the normalized attribute value, the best value is 1 , and the worst value is 0 .
(3) Computing node quality $M_{i}$

$$
M_{i}=\frac{1}{m} \sum_{j=1}^{m} w_{j} z_{i j}, i=1,2, \ldots, n, \sum_{i=1}^{m} w_{i}=1
$$

Step2: Computing the topological potential of each node $\varphi\left(v_{i}\right), i=1,2, \ldots, n$

Get $M_{i}$ into Eq.1 to compute $\varphi\left(v_{i}\right)$.

Step3: Sorting node importance by comparing the value of topological potential

Sort node importance according to the size of topological potential, and the greater the topological potential, the more importance in network.

\section{EXPERIMENT AND ANALYSIS}

As shown in Fig.1, a propagation network of public opinion contains 10 nodes and 9 edges, where each node represents the participants in public opinion. Node's attribute values as shown in Table 2 .

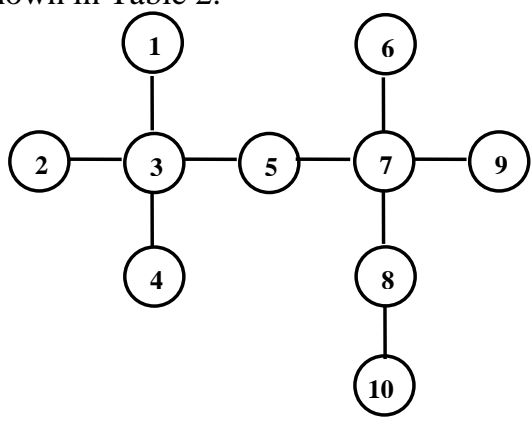

Figure 1. A simple opinion network

TABLE II. ATtRIBUte VAlUES

\begin{tabular}{|c|c|c|c|c|}
\hline & $X_{1}$ & $X_{2}$ & $X_{3}$ & $X_{4}$ \\
\hline$v_{1}$ & 6 & 2 & 8 & 48 \\
\hline$v_{2}$ & 8 & 4 & 9 & 80 \\
\hline$v_{3}$ & 4 & 1 & 7 & 45 \\
\hline$v_{4}$ & 9 & 20 & 10 & 120 \\
\hline$v_{5}$ & 1 & 8 & 5 & 55 \\
\hline$v_{6}$ & 5 & 0 & 6 & 20 \\
\hline$v_{7}$ & 9 & 10 & 7 & 108 \\
\hline$v_{8}$ & 0 & 4 & 5 & 80 \\
\hline$v_{9}$ & 2 & 2 & 9 & 95 \\
\hline$v_{10}$ & 8 & 4 & 6 & 76 \\
\hline
\end{tabular}


Normalizing the node attribute value following Eq.1, the results are shown in Table 3.

TABLE III. THE NORMALIZED ATTRIBUTE VALUES

\begin{tabular}{|c|c|c|c|c|}
\hline & $\begin{array}{c}\text { Debate } \\
Z_{1}\end{array}$ & $\begin{array}{c}\text { Influence } \\
Z_{2}\end{array}$ & $\begin{array}{c}\text { Obstinacy } \\
Z_{3}\end{array}$ & $\begin{array}{c}\text { Activity } \\
Z_{4}\end{array}$ \\
\hline$v_{1}$ & 0.6667 & 0.1000 & 0.6000 & 0.2800 \\
\hline$v_{2}$ & 0.8889 & 0.2000 & 0.8000 & 0.6000 \\
\hline$v_{3}$ & 0.4444 & 0.0500 & 0.4000 & 0.2500 \\
\hline$v_{4}$ & 1.0000 & 1.0000 & 1.0000 & 1.0000 \\
\hline$v_{5}$ & 0.1111 & 0.4000 & 0.0000 & 0.3500 \\
\hline$v_{6}$ & 0.5556 & 0.0000 & 0.2000 & 0.0000 \\
\hline$v_{7}$ & 1.0000 & 0.5000 & 0.4000 & 0.8800 \\
\hline$v_{8}$ & 0.0000 & 0.2000 & 0.0000 & 0.6000 \\
\hline$v_{9}$ & 0.2222 & 0.1000 & 0.8000 & 0.7500 \\
\hline$v_{10}$ & 0.8889 & 0.2000 & 0.2000 & 0.5600 \\
\hline
\end{tabular}

TABLE IV. The VAlues SET FOR JUdgment

\begin{tabular}{|c|c|c|}
\hline $\begin{array}{c}\text { Relative } \\
\text { importance } \\
\text { degree }\end{array}$ & Definition & Explain \\
\hline 1 & Equally important & $\begin{array}{c}\text { Two attributes are equally } \\
\text { important }\end{array}$ \\
\hline 3 & Slightly important & $\begin{array}{c}\text { A attribute slightly important } \\
\text { than another }\end{array}$ \\
\hline 5 & Quite important & $\begin{array}{c}\text { A attribute important than } \\
\text { another }\end{array}$ \\
\hline 7 & $\begin{array}{c}\text { Obvious } \\
\text { important }\end{array}$ & $\begin{array}{c}\text { A attribute obviously important } \\
\text { than another }\end{array}$ \\
\hline 9 & $\begin{array}{c}\text { Absolutely } \\
\text { important }\end{array}$ & $\begin{array}{c}\text { A attribute deeply important } \\
\text { than another }\end{array}$ \\
\hline $2,4,6,8$ & $\begin{array}{c}\text { Intermediate } \\
\text { values }\end{array}$ & Need to compromise \\
\hline
\end{tabular}

In order to compare the relative importance between attributes, we get $b_{i j}$ with reference to the level table given by Saaty, which are shown in Table $4 . b_{i j}$ denotes the relative importance from the i-th attribute to the j-th attribute, and we think that this is the ratio of the weight $w_{i}$ of attribute $i$ to the weight $w_{j}$ of attribute $j$.

$$
b_{i j}=\frac{w_{i}}{w_{j}}
$$

According to the experience about public opinion research, we can think that the debate $X_{1}$ compared with the influence $X_{2}$ between "equally important" and "slightly important”, therefore $b_{12}=2$. Without loss of generality, this paper compare the importance of each attribute in pairs and according to Eq.4 get the attribute relative importance judgment matrix $B[6]$ :

$$
B=\left[\begin{array}{cccc}
1 & 2 & 3 & 7 \\
1 / 2 & 1 & 1 & 5 \\
1 / 3 & 1 & 1 & 5 \\
1 / 7 & 1 / 5 & 1 / 5 & 1
\end{array}\right]
$$

According to Eq.5 and $\sum_{i=1}^{m} w_{i}=1$ are $m+1$ equations, where $w_{1}, w_{2}, \ldots, w_{m}$ and $\lambda$ have $m+1$ variables, therefore the weights of attributes are $w=[0.5037,0.2461,0.1922,0.0579]^{T}$.

$$
\begin{gathered}
\sum_{i=1}^{m}\left(b_{i l}-w_{i}\right) b_{i l}-\sum_{j=1}^{m}\left(b_{l j}-w_{l}\right)+\lambda=0 \\
l=1,2, \ldots, m .
\end{gathered}
$$

Then we compute each node quality according to Eq.3. The results are as follows: $M_{1}=0.1230$, $M_{2}=0.1714, M_{3}=0.0980, M_{4}=0.2500$, $M_{5}=0.0437, M_{6}=0.0796, M_{7}=0.1886$, $M_{8}=0.0210, M_{9}=0.0834, M_{10}=0.1420$.

The impact factor $\sigma$ in the formula of topological potential is used to control the effect area of each node. Usually we can optimize $\sigma$ according to the potential entropy of nodes in network. In this paper we choose $\sigma=1.374$.

Therefore according to the Eq.1, we compute topological potential $\varphi\left(v_{i}\right)$. The comparison between some evaluation methods and topological potential are shown in Table 5.

TABLE V. The EVALUATION VALUES OF NODE IMPORTANCE

\begin{tabular}{|c|c|c|c|c|c|}
\hline Node & Degree & $\begin{array}{c}\text { Betwe- } \\
\text { enness }\end{array}$ & $\begin{array}{c}\text { Close- } \\
\text { ness }\end{array}$ & $\begin{array}{c}\text { Simplified } \\
\text { Topological } \\
\text { Potential }\end{array}$ & $\begin{array}{c}\text { Topological } \\
\text { Potential }\end{array}$ \\
\hline$v_{1}$ & 1 & 0 & 0.037 & 0.192 & 0.238 \\
\hline$v_{2}$ & 1 & 0 & 0.037 & 0.192 & 0.281 \\
\hline$v_{3}$ & 4 & 0.468 & 0.053 & 0.344 & 0.469 \\
\hline$v_{4}$ & 1 & 0 & 0.037 & 0.192 & 0.350 \\
\hline$v_{5}$ & 2 & 0.444 & 0.059 & 0.283 & 0.301 \\
\hline$v_{6}$ & 1 & 0 & 0.040 & 0.193 & 0.211 \\
\hline$v_{7}$ & 4 & 0.556 & 0.059 & 0.356 & 0.356 \\
\hline$v_{8}$ & 2 & 0.178 & 0.044 & 0.250 & 0.241 \\
\hline$v_{9}$ & 1 & 0 & 0.040 & 0.193 & 0.214 \\
\hline$v_{10}$ & 1 & 0 & 0.032 & 0.171 & 0.179 \\
\hline
\end{tabular}


From the experiment results, we can reach a conclusion that this method presented in this paper can more accurate shows the node's position information and difference in network. Comprehensive consideration for the node's properties and the network topology will more close to the actual situation than pure consideration on the network topology. We find that from the experiment results, node $v_{7}$ is the most important node by using some traditional evaluation methods, but if we still consider the comprehensive attributes of nodes, node $v_{4}$ more important than node $v_{7}$. Node $v_{1}, v_{2}$ and $v_{4}$, node $v_{6}$ and $v_{9}$ seem to be completely symmetrical structure in the network topology, so they will also have the same importance according to the evaluation indexes of topological structure. But in the public opinion network the nodes represent the participants of topic, they have differences take for granted, which abstract perform to be the different attributes of nodes. Therefore, node $v_{4}$ is more important than node $v_{2}$, node $v_{2}$ is more important than node $v_{1}$, and node $v_{9}$ is more important than node $v_{6}$.

Total time complexity of algorithm depends on the calculation of topological potential, it is $O\left(n^{2}\right)$ in the worst case and $O\left(m+n^{3 / \gamma}\right)$ in the best case, where $n$ nodes, $m$ edges and $2<\gamma<3$ denotes a constant.

\section{SUMMARY}

The research about node importance in public opinion is helpful to reasonable and effective monitor and forecast the development and change of public opinion, exact find out those nodes which have important influence to the spread of public opinion in the complex environment. The monitoring and technical processing of important nodes can very effective guide public opinion, at the same time they have the important meaning to create a healthy and orderly network environment for public opinion and the harmonious society. This paper summarizes the research about evaluation of node importance, references the idea of topological potential in physics and establishes the evaluation algorithm of node importance in public opinion network. This method takes into account the network topology and node's attributes, so it can more accurate evaluate node importance. Through the theoretical and experimental analysis, it is proved that this method is significant both to theory and practice.

\section{ACKNOWLEDGMENT}

This research is supported by the Opening Foundation of Key Laboratory of Pattern Recognition and Intelligent Information Processing, Institutions of Higher Education of Sichuan Province, Chengdu University and by the Funds of Chengdu Normal University of China (Grants Nos. CS14ZD03, YJRC2015-7).

\section{REFERENCES}

[1] Payne S.L.B, The Art of Asking Questions: Studies in Public Opinion, Princeton University Press, 2014.

[2] Hu P, Fan W, Mei S, Identifying node importance in complex networks, Physica A. 429 (2015) 169-176.

[3] Burt R.S, Kilduff M, Tasselli S, Social network analysis: Foundations and frontiers on advantage, Annual review of psychology. 64 (2013) 527-547.

[4] Lü L, Zhou T, Zhang Q.M, et al, The H-index of a network node and its relation to degree and coreness, Nature communications. 7 (2016).

[5] Wu Y, Yang Y, Jiang F, et al, Coritivity-based influence maximization in social networks, Physica A. 416 (2014) 467-480.

[6] Suo S, Chen Y, The dynamics of public opinion in complex networks, Journal of Artificial Societies and Social Simulation. 11 (2008) 2.

[7] Zhang Y, Gu L, Liao X, et al, FRANK: A Fast Node Ranking Approach in Large-Scale Networks, IEEE Network. 31 (2017) 36-43.

[8] Han R, Feng C, Xia H, Optimal user association based on topological potential in heterogeneous networks, 2013 IEEE 24th International Symposium on IEEE. (2013)

[9] Dong Z, Long G, Virtual network mapping through locality-aware topological potential and influence node ranking, Chinese Journal of Electronics. 23 (2014).

[10] Lu H.Z, Zhang S.B, Shen S.Q. High-field magnetoconductivity of topological semimetals with short-range potential, Physical Review B. 92 (2015) 045203. 